\title{
Lenalidomide-Related Spinal Cord Infarction in Primary
}

\section{Amyloidosis}

\author{
Chen Makranz ${ }^{*}$, Boaz Nachmias ${ }^{2}$, Moshe E. Gatt ${ }^{2}$, Ruth Eliahou ${ }^{3}$, Alexander Lossos ${ }^{1}$ \\ ${ }^{1}$ Departments of Oncology and Neurology, Leslie and Michael Gaffin Center for Neuro-Oncology, Hadassah- \\ Hebrew University Medical Center, Jerusalem, Israel \\ ${ }^{2}$ Department of Hematology, Hadassah-Hebrew University Medical Center, Jerusalem, Israel \\ ${ }^{3}$ Department of Radiology, Hadassah-Hebrew University Medical Center, Jerusalem, Israel
}

"Corresponding Author: Dr. Chen Makranz, Departments of Oncology and Neurology, Leslie and Michael Gaffin Center for Neuro-Oncology, Hadassah-Hebrew University Medical Center, Jerusalem, 91120, Israel, Tel: +972-0505172614; Fax: 972-2-6437782; E-mail: chenmak@ hadassah.org.il

Received: 18 July 2018; Accepted: 31 July 2018; Published: 02 August 2018

\begin{abstract}
Spinal cord infarction is a rare but often devastating disorder caused by disruption in the vascular spinal supply. We report a patient with spinal cord infarction associated with lenalidomide therapy for primary amyloidosis, in the background of other predisposing factors. We describe the clinical case and discuss the possible explanation for this rare complication. As spinal cord infarction is not a known complication of lenalidomide therapy or amyloidosis, this description raises the potential of such a side effect in some certain conditions and discusses the role of the old and new anticoagulant agents in prevention of thromboembolic complications in high-risk patients.
\end{abstract}

Keywords: Amyloidosis; Enoxaparin; Lenalidomide; Spinal cord infarction; Thrombosis

\section{Introduction}

Spinal cord infarction is a rare but often devastating disorder, accounting for only $1 \%$ of all strokes and caused by disruption in the vascular spinal supply, most commonly the anterior spinal artery. Typical presentation includes acute paraparesis or quadriparesis, depending on the involved spinal cord level. Posterior spinal artery infarction is less frequent and causes mainly sensory deficit. The diagnosis requires neuroimaging confirmation and determination of the underlying etiology, mainly prolonged arterial hypoperfusion and mechanical stress by disc 
prolapse. However most cases were idiopathic [1-2]. We report for the first time a patient with primary AL amyloidosis who developed spinal cord infarction associated with lenalidomide therapy in the background of other risk factors and possibly sub-optimal thromboprophylaxis.

\section{Case Report}

A 72- years-old man with primary AL Lambda light chain amyloidosis of 5 years duration presented with acute left leg monoplegia accompanied by hypoesthesia and urinary incontinence without back pain. He was a smoker and had known right leg weakness related to poliomyelitis in childhood. Manifestations of amyloidosis included skeletal amyloidomas, renal (proteinuria, $700 \mathrm{mg} / 24 \mathrm{~h}$ ) and cardiac involvement with intraventricular septum hypertrophy and mild cardiac failure. Four years earlier, he had spinal cord compression with left leg flaccid paralysis and right leg hypoesthesia, related to a T4 vertebral biopsy proven amyloidoma. He was treated with irradiation (T3-T10 bodies, $3 \mathrm{~Gy} \times 10$ ) and a few months later were able to move his legs against gravity. He was also treated with melphalan, prednisone and thalidomide, discontinued because of peripheral neuropathy and replaced by melphalan, dexamethasone and lenalidomide for a year. Following 2 years of complete remission, he had a systemic relapse manifested by light chain elevation and proteinuria, and lenalidomide was reintroduced in combination with dexamethasone. While on lenalidomide, he developed left calf deep vein thrombosis (DVT) treated with enoxaparin $60 \mathrm{mg}$ twice a day, later reduced to $40 \mathrm{mg}$ once a day because of recurrent hematuria. A week before the current admission, enoxaparin was replaced by low dose rivaroxaban (10 $\mathrm{mg}$ once a day) due to renal failure with a creatinine clearance of $\sim 40$ and nacrohematuria.

On examination, he had a complete paresis of the proximal left leg, a mild left foot weakness, and hypoesthesia in the L4-L5 distribution on the left side, in addition to the known flaccid paralysis of the right leg, attributed to past poliomyelitis. Spinal computed tomography scan (CT) showed cervical and mid-thoracic vertebral lytic lesions with no evidence of cord or root compression.

Magnetic resonance imaging (MRI) showed a non-enhancing T2-hyperintense intramedullary lesion and restricted diffusion at T11-L1 without atrophy or swelling, compatible with an acute spinal cord infarction (Figure 1). Diagnosis of delayed post-radiation myelopathy was raised but considered less likely because of absent matching radiation field vertebral bone marrow changes, absent contrast enhancement and edema, absent cord atrophy, long latent interval since the previous radiotherapy, and the presence of restricted diffusion [3]. Hypercoagulability profile and echocardiogram, including bubble study to rule out cardiac thrombi and right-to-left shunt, were negative. Rivaroxaban levels were not available at that time. However, international normalized ratio (INR) was 1.6 (normal values 1-1.4) and partial thromboplastin time (PTT) was 30.6 seconds (normal values $24-40$ seconds). After ruling out alternative causes, we suspected lenalidomide-related spinal cord infarction, possibly combined with insufficient thromboprophylaxis on rivaroxaban. Therefore, lenalidomide and rivaroxaban were discontinued and enoxaparin with aspirin were initiated, followed in the next few weeks by gradual improvement of the left leg weakness. One month later, lenalidomide was re-introduced with no further complications. 


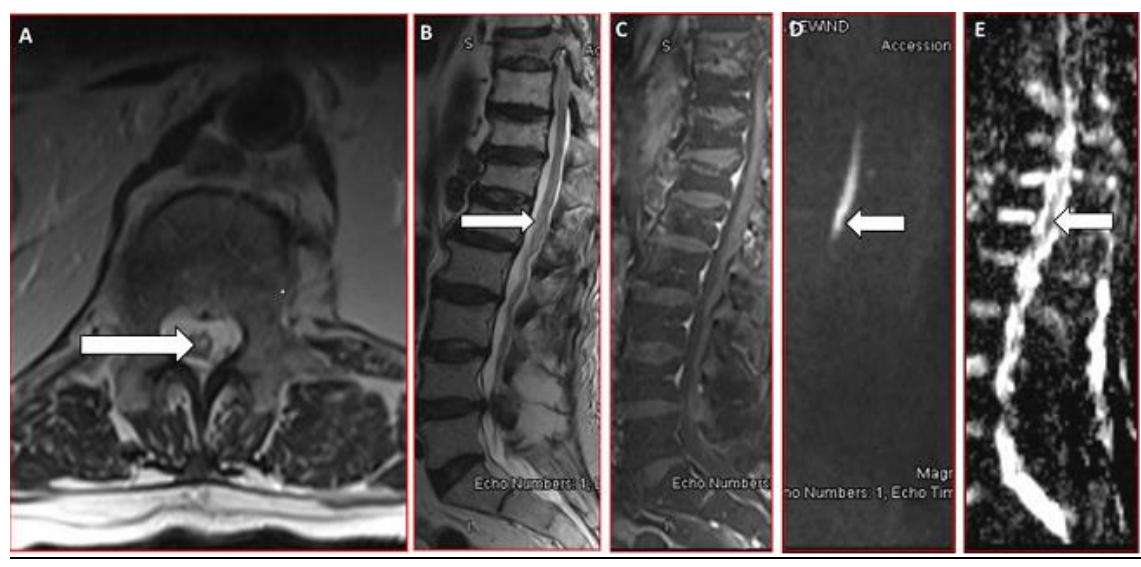

Figure 1: Neuororadiological diagnosis of spinal cord infarction on admission. Axial (A) and sagittal (B) Lumbar spine MRI shows T2-hyperinstense intramedullary central signal abnormality at the T11-L1 levels with no atrophy or swelling of the cord, without gadolinium enhancement (C). Central abnormal restricted diffusion on diffusionweighted (DWI) image (D) also shown in the corresponding apparent diffusion coefficient (ADC) image (E), suggesting acute spinal cord ischemia.

\section{Discussion}

Our patient presented with acute left leg weakness while on lenalidomide for primary AL amyloidosis. The clinical presentation, together with the imaging findings strongly suggested acute spinal cord infarction at a previously nonirradiated T11-L1 segment.

Lenalidomide is an immunomodulatory (IMiD) derivative of thalidomide that down-regulates interleukin-6 and nuclear factor $\kappa-\mathrm{B}$, and activates caspase 8 in vitro [4], with a known prothrombotic activity due to tumor necrosis factor alpha $(\mathrm{TNF} \alpha)$ upregulation, endothelial cell dysfunction and thrombospondin downregulation leading to a cytokine-mediated and activated protein $\mathrm{C}$ resistance [5]. Reported lenalidomide-related thromboembolic complications are mostly venous, such as DVT and pulmonary embolism, while arterial events such as cerebrovascular ischemia are less common. Spinal arterial ischemia was not reported previously in association to lenalidomide. Similar to thalidomide, the risk for thrombotic complications is increased when treatment is combined with corticosteroids or chemotherapy, and thromboprophylaxis decreases their incidence [6-7]. While low molecular weight heparin (LMWH) was proven to be effective in prevention of IMid related thromboembolism [8], the use of direct oral anticoagulants (DOACs) in prevention of IMiD related thromboembolism is not well established. A small retrospective series [9] found it to be safe and effective; however, it is still investigational and should be reconsidered in high risk patients. Our case again demonstrates the risk in treatment with DOACs for thromboprophylaxis in high risk patients on IMiDs.

Our patient had primary AL amyloidosis, implicated in thrombogenesis through several mechanisms. Hypercoagulability in amyloidosis is induced by nephrotic syndrome resulting in imbalance of antithrombotic and prothrombotic factors involved in the coagulation cascade [10]. Cardiac thrombi are caused by restrictive 
cardiomyopathy and frequent arrhythmia due to the amyloid deposition [11]. However, neither arrhythmia nor thrombi were found in our patient. Some patients also develop hyposplenism with thrombocytosis or, infrequently, blood hyperviscosity or circulating monoclonal procoagulant activity [11], which are also irrelevant in our patient. Therapeutic immunomodulation may further add thrombotic complications [6-7]. Most ischemic events in primary amyloidosis are cerebrovascular of cardioembolic source, while peripheral arterial, mesenteric and optic nerve events are less common [11]. Spinal cord infarction was not reported, to our knowledge, in this clinical setup.

Our patient had a T11-L1 spinal cord infarction, an uncommon location among ischemic spinal events. This segment is supplied by a branch of the intercostal artery arising between T8 and L1, called Adamkiewicz artery [2]. Since previous radiation fields in our patient included these segments, it is possible that Adamkiewicz artery was exposed, predisposing it to radiation-induced accelerated atherosclerosis [12] with a risk for ischemia in its territory. Because no spinal angiogram was performed, we could not confirm this possibility. It should be noted that our patient was a smoker, thus rendering him high risk for arterial occlusion and thrombosis. However, spinal cord ischemia was reported to be rare even among patients with cardiovascular risk factors [13].

Taken together, it seems likely that our patient manifested lenalidomide-related spinal cord infarction on the background of multiple predisposing factors, including smoking, AL amyloidosis, and, possibly, accelerated radiation-induced atherosclerosis, combined with sub-optimal thromboprophylaxis due to exchanging enoxaparin with rivaroxaban. Re-treatment with lenalidomide after the patient had been well anticoagulated by enoxaparin was proven to be safe, hence emphasizes the importance of thromboprophylaxis in the context of IMid therapy.

\section{Conclusion}

Spinal cord infarction is a rare ischemic manifestation, even among patients with cardiovascular risk factors. It was not previously reported in relation to neither amyloidosis nor lenalidomide treatment. However, this rare complication should be considered in the differential diagnosis in the appropriate clinical context among patients treated by lenalidomide, especially when other risk factors co-exist. In the face of the widespread use of DOACs, we suggest that for high risk patients receiving lenalidomide, LMWH should still be considered as the optimal agent for thromboprophylaxis.

\section{Acknowledgements}

Corresponding author has nothing to declare; this research did not receive any specific grant from funding agencies in the public, commercial, or not-for-profit sectors.

\section{Conflict of Interest}

None 


\section{References}

1. Nogueira RG, Ferreira R, Grant PE, et al. Restricted diffusion in spinal cord infarction demonstrated by magnetic resonance line scan diffusion imaging. Stroke 43 (2012): 532-535.

2. Novy J, Carruzzo A, Maeder P, et al. Spinal cord ischemia: clinical and imaging patterns, pathogenesis, and outcomes in 27 patients. Arch Neurol 63 (2006): 1113-1120.

3. Komachi H, Tsuchiya K, Ikeda M, et al. Radiation telepathy: A clinicopathological study with special reference to correlation between MRI findings and neuropathology. Journal of the Neurological Sciences 132 (1995): 228-232.

4. Hideshima T, Chauhan D, Shima Y, et al. Thalidomide and its analogs overcome drug resistance of human multiple myeloma cells to conventional therapy. Blood 96 (2000): 2943-2950.

5. Aue G, Lozier JN, Tian X, et al. Inflammation, TNF $\alpha$ and endothelial dysfunction link lenalidomide to venous thrombosis in chronic lymphocytic leukemia. American Journal of Hematology 86 (2011): 835-840.

6. Menon SP, Rajkumar SV, Lacy M, et al. Thromboembolic events with lenalidomide-based therapy for multiple myeloma. Cancer 112 (2008): 1522-1528.

7. Tannemaat MR, Vries EP, Molendijk WJ, et al. Fatal ischemic stroke in a patient receiving lenalidomide for multiple myeloma. Clin Neurol Neurosurg 113 (2011): 488-489.

8. Khorana AA, Carrier M, Garcia DA, et al. Guidance for the prevention and treatment of cancer-associated venous thromboembolism. Journal of Thrombosis and Thrombolysis 41 (2016): 81-91.

9. Man L, Morris AL, Brown J, et al. Use of Direct Oral Anticoagulants in Patients on Immunomodulatory Agents. Blood 128 (2016): 1449.

10. Bever KM, Masha LI, Sun F, et al. Risk factors for venous thromboembolism in immunoglobulin light chain amyloidosis. Haematologica 101 (2016): 86-90.

11. Hausfater $\mathrm{P}$, Costedoat-Chalumeau N, Amoura Z, et al. AL cardiac amyloidosis and arterial thromboembolic events. Scandinavian Journal of Rheumatology 34 (2005): 315-319.

12. McGuirt WF, Feehs RS, Strickland HL, et al. Irradiation-Induced Atherosclerosis: A Factor in Therapeutic Planning. Annals of Otology, Rhinology \& Laryngology 101 (1992): 222-228.

13. Romi F, Naess H. Spinal Cord Infarction in Clinical Neurology: A Review of Characteristics and LongTerm Prognosis in Comparison to Cerebral Infarction. Eur Neurol 76 (2016): 95-98.

Citation: Chen Makranz, Boaz Nachmias, Moshe E. Gatt, Ruth Eliahou, Alexander Lossos. Lenalidomide-Related Spinal Cord Infarction in Primary Amyloidosis. Archives of Clinical and Medical Case Reports 2 (2018): 124-128.

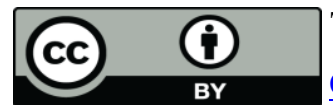

This article is an open access article distributed under the terms and conditions of the Creative Commons Attribution (CC-BY) license 4.0 